\title{
Benign familial infantile convulsions: a clinical study of seven Dutch families
}

\section{PETRA MC CALLENBACH, ${ }^{1}$ RENÉ FM DE COO ${ }^{2}$ ALLA A VEIN, ${ }^{1}$ WILLEM FRANS M ARTS ${ }^{2}$ JAN C OOSTERWIJK, ${ }^{3}$ GERARD HAGEMAN, ${ }^{4}$ ROBERT TEN HOUTEN, ${ }^{5}$ GISELA M TERWINDT, ${ }^{1}$ DICK LINDHOUT, ${ }^{6}$ RUNE R FRANTS, ${ }^{7}$ OEBELE F BROUWER ${ }^{1,8}$}

\author{
${ }^{1}$ Department of Neurology and Clinical Neurophysiology, Leiden University Medical Centre, Leiden; ${ }^{2}$ Department of \\ Paediatric Neurology, Erasmus Medical Centre Rotterdam, Rotterdam; ${ }^{3}$ Department of Medical Genetics, University \\ Hospital Groningen, Groningen; ${ }^{4}$ Department of Neurology, Medical Spectrum Twente, Enschede; ${ }^{5}$ Department of \\ Neurology, Medical Centre Alkmaar, Alkmaar; ${ }^{6}$ Department of Medical Genetics, University Medical Centre Utrecht, \\ Utrecht; ${ }^{7}$ Department of Clinical and Human Genetics, Leiden University Medical Centre, Leiden; ${ }^{8}$ Department of \\ Neurology, University Hospital Groningen, Groningen, The Netherlands
}

Benign familial infantile convulsions (BFIC) is a recently identified partial epilepsy syndrome with onset between 3 and 12 months of age. We describe the clinical characteristics and outcome of 43 patients with BFIC from six Dutch families and one Dutch-Canadian family and the encountered difficulties in classifying the syndrome. Four families had a pure BFIC phenotype; in two families BFIC was accompanied by paroxysmal kinesigenic dyskinesias; in one family BFIC was associated with later onset focal epilepsy in older generations. Onset of seizures was between 6 weeks and 10 months, and seizures remitted before the age of 3 years in all patients with BFIC. In all, 29 (67\%) of the 43 patients had been treated with anti-epileptic drugs for a certain period of time.

BFIC is often not recognized as (hereditary) epilepsy by the treating physician. Seizures often remit shortly after the start of anti-epileptic drugs but, because of the benign course of the syndrome and the spontaneous remission of seizures, patients with low seizure frequency do not necessarily have to be treated. If prescribed, anti-epileptic drugs can probably be withdrawn after 1 or 2 years of seizure freedom.

Keywords: Benign familial infantile convulsions. Epilepsy. Paroxysmal kinesigenic dyskinesias. Genetics.

\section{Introduction}

Benign familial infantile convulsions (BFIC) is a recently identified partial epilepsy syndrome of early childhood, characterized by brief seizures with motor arrest, slow deviation of the head and eyes to one side, generalized hypertonia, cyanosis, and unilateral limb jerks becoming bilateral, synchronous or asynchronous. ${ }^{1-8}$ The age at onset is between 3 and 12 months and seizures may occur in clusters of 2-4 days. Anti-epileptic drugs (AEDs) are very effective, and seizures remit before the age of 3 years. The ictal electroencephalogram (EEG) shows parieto-occipital epileptic activity that may become generalized; most interictal EEGs show no abnormalities, but sometimes few slow spikewaves can be observed. Subsequent psychomotor development is normal. BFIC syndrome is autosomal dominantly inherited with incomplete penetrance. Three loci have been described: in five Italian families linkage was observed with chromosome ${ }^{19} q,{ }^{9}$ in seven families from France and Argentina with chromosome 16p12-q12,10 and in another eight Italian families with chromosome 2q24. ${ }^{11}$ No phenotypic differences could be

Received 22.02.02. Revised 10.06.02. Accepted 02.07.02.

Correspondence: OF Brouwer, Department of Neurology, University Hospital Groningen, PO Box 30001, 9700 RB Groningen, The Netherlands; Tel: +50-3612430; Fax: +50-3611707; e-mail: o.f.brouwer@neuro.azg.nl 
observed between families linked to different chromosomes.

In some families, patients with BFIC also experience paroxysmal dyskinesias at a later age, with onset in childhood or early adolescence. These dyskinesias include unilateral or bilateral choreoathetosis and dystonia that may occur spontaneously or be kinesigenic, i.e. induced by sudden voluntary movements. ${ }^{12-15}$ Many patients report variable sensations preceding an attack. The dyskinesias usually last a few seconds, during which consciousness is preserved, and are easily controlled by AEDs. Frequency and severity usually decline with age. This syndrome has been identified as infantile convulsions and choreoathetosis (ICCA). ICCA has been mapped to chromosome 16p12-q12, the same region as has been identified in the above-mentioned French and Argentinian families with BFIC. ${ }^{\mathbf{1 2}-15}$ The responsible gene that is located in this area might, apparently, cause both BFIC with and without paroxysmal kinesigenic dyskinesias.

We describe 43 patients with BFIC from six Dutch families and one Dutch-Canadian family. Four families had a pure BFIC phenotype (families 1, 4, 6 and 7), in two families BFIC was accompanied by paroxysmal kinesigenic dyskinesias (families 3 and 5), and in one family BFIC was associated with later onset focal epilepsy in older generations (family 2). The Dutch-Canadian family, in which the epilepsy shows partial cosegregation with hemiplegic migraine, has been described elsewhere. ${ }^{16}$ The aim of this study is to delineate the phenotype and outcome of BFIC. Furthermore, we performed linkage analysis in the largest family (7) with the three known loci for BFIC. ${ }^{9-11}$

\section{Patients}

The described families are included in a nationwide study of the genetics of idiopathic epilepsies in the Netherlands. After obtaining written informed consent, clinical characteristics of the seizures of all participating affected relatives and, if performed, results of EEGs, cerebral computed tomography $(\mathrm{CT})$, and magnetic resonance imaging (MRI) were obtained from the treating physician. If the acquired information was insufficient to classify the seizures, patients and their parents were interviewed. Seizures were classified according to the criteria of the International League Against Epilepsy. ${ }^{17}$

The diagnosis BFIC was made if (1) focal afebrile seizures without known causative factors occurred that could become secondary generalized; (2) onset of seizures was between the age of 1 month and 1 year; (3) seizures remitted spontaneously at young age; (4) children were otherwise healthy; and (5) a family history of similar seizures existed. ${ }^{2,3}$

This study has been approved by the medical ethical board of the Leiden University Medical Centre and the Erasmus Medical Centre Rotterdam.

\section{Results}

We included seven families in which at least two individuals in one generation were affected with seizures that fulfilled the criteria of benign infantile convulsions (Fig. 1). In total, 51 persons (had) suffered from epilepsy. Clinical information about four affected persons could not be obtained because they were deceased (two cases) or did not want to participate (two cases), so these persons are not included in this article. Of the 47 described patients with epilepsy, four did not suffer from infantile convulsions but from focal epilepsy with a different age at onset: three had epilepsy with a later age at onset (family 2, II:1, III:1, and III:10), and one had neonatal convulsions (family 4, III:3). Detailed clinical descriptions of all affected relatives are given in the appendix at the end of this article. The families showed autosomal dominant inheritance with high penetrance. Families 2 and 7 showed incomplete penetrance that could be due to the fact that the oldest generation was deceased, so that accurate parental information about the infantile period of the second generation was missing. Four families had a pure BFIC phenotype (families 1, 4, 6 and 7), in two families BFIC was accompanied by paroxysmal kinesigenic dyskinesias (families 3 and 5), and, as mentioned earlier, BFIC was associated with later onset focal epilepsy in older generations in one other family (family 2). Four affected relatives of family 6 also had hemiplegic migraine. This family has been described earlier by Terwindt et al. ${ }^{16}$

Onset of seizures ranged from 6 weeks to approximately 10 months, and seizures remitted before the age of 3 years in all 43 patients with BFIC (Table 1, Fig. 2). Accurate information about the age at onset and remission and about the period of AEDs treatment could often not be obtained for the older generations: medical files had already been destroyed and we were, therefore, dependent on the recollection of the symptoms by the parents. Seizures were tonic, tonic-clonic or atonic, and were often accompanied by unresponsiveness, gazing, cyanosis, and deviation of head and eyes. 

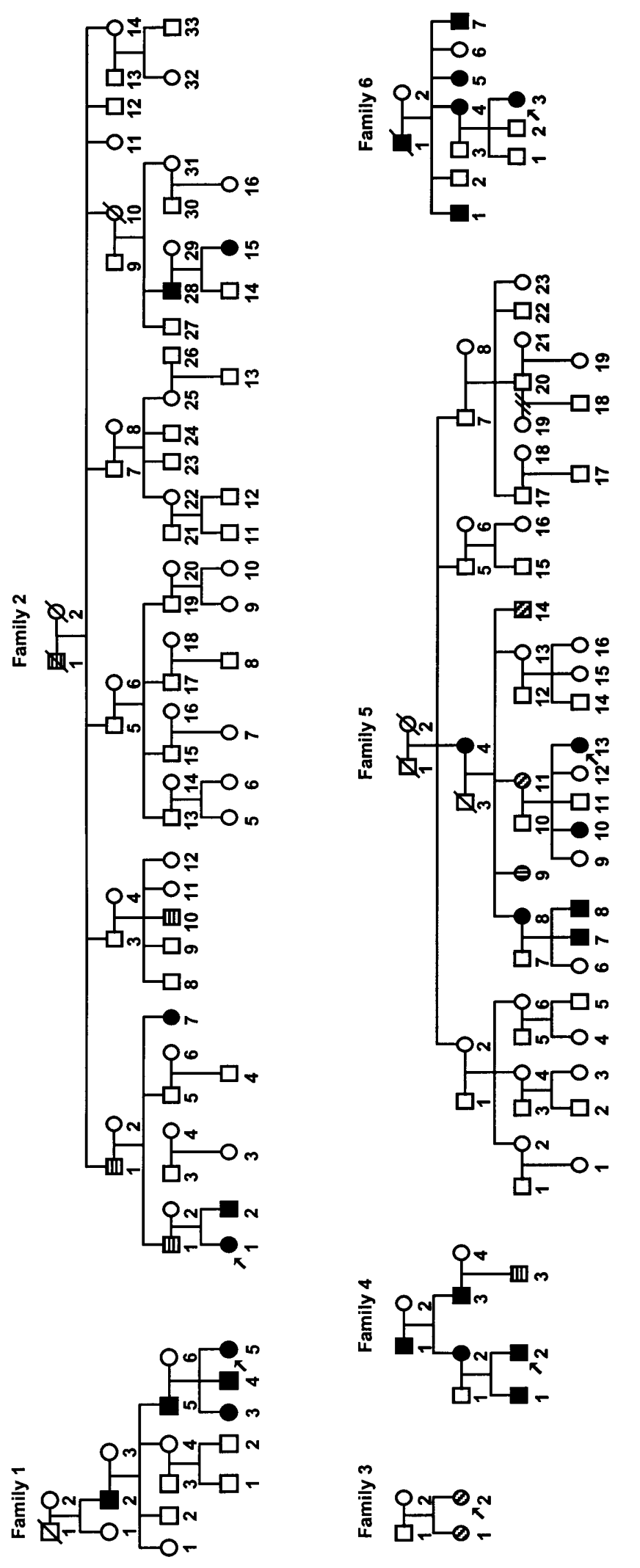
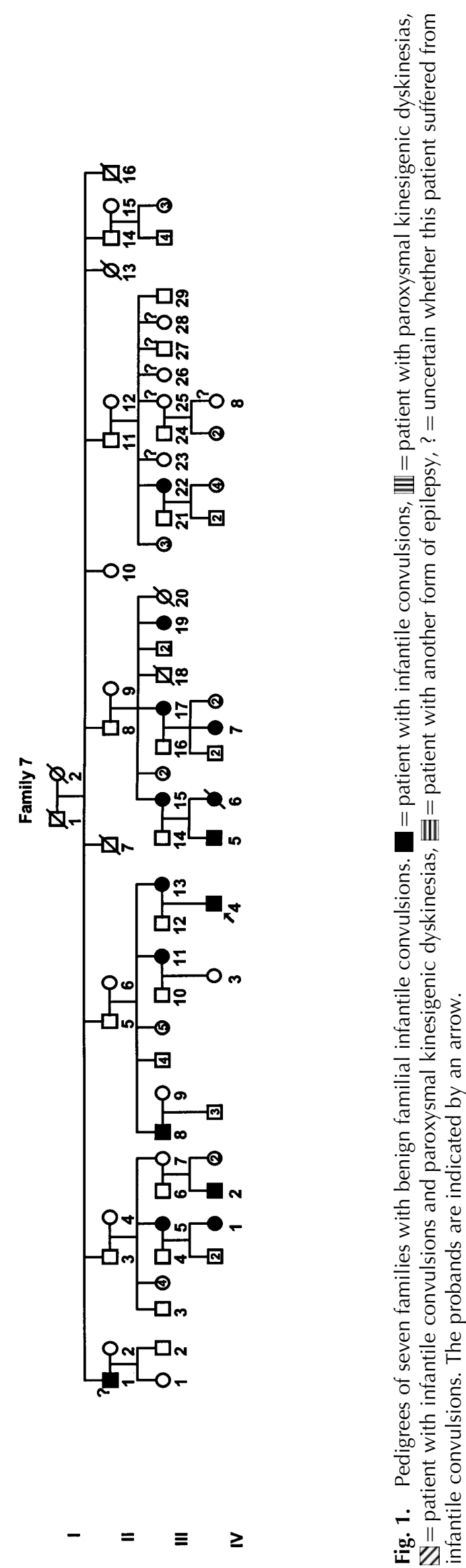
Table 1. Clinical characteristics of relatives with epilepsy

\begin{tabular}{|c|c|c|c|c|c|c|c|c|c|}
\hline & & & & Age & & & & & \\
\hline & ient & Sex & $\begin{array}{l}\text { Onset } \\
\text { (months) }\end{array}$ & $\begin{array}{l}\text { Remission } \\
\text { (months) }\end{array}$ & $\begin{array}{l}\text { Current } \\
\text { (years) }\end{array}$ & AEDs & Seizure classification & Other & Diagnosis \\
\hline 1 & II:2 & $\mathrm{M}$ & 9.0 & 36 & 69 & $?$ & GTCS & & BFIC \\
\hline & III:5 & $\mathrm{M}$ & 6.0 & 36 & 31 & PHB & GTCS & & BFIC \\
\hline & IV:3 & $\mathrm{F}$ & 5.9 & 7.8 & 5 & none & atonic & & BFIC \\
\hline & IV:4 & $\mathrm{M}$ & 6.0 & 6.2 & 4 & VPA & $\begin{array}{l}\text { tonic, atonic, jerks around } \\
\text { mouth }\end{array}$ & & BFIC \\
\hline & IV:5 & $\mathrm{F}$ & 4.7 & 5.1 & 0.8 & VPA & focal motor, $1 \times$ GTCS & & BFIC \\
\hline 2 & II:1 & $\mathrm{M}$ & $\begin{array}{l}17 \text { yrs+ } \\
59 \text { yrs }\end{array}$ & a & 69 & $\begin{array}{l}\text { PHT/VPA/ } \\
\text { LTG }\end{array}$ & atonic/GTCS, CPS & & foc. cryp. \\
\hline & III:1 & $\mathrm{M}$ & 31 yrs & 31.2 yrs & 38 & none & TCS & & $?$ \\
\hline & III:7 & $\mathrm{F}$ & 8.5 & 31 & 30 & $\mathrm{PHB}+\mathrm{PHT}$ & GTCS & & BFIC \\
\hline & III:10 & $\mathrm{M}$ & 16.7 yrs & 18.8 yrs & 25 & $\mathrm{CBZ}+\mathrm{VGB}$ & focal motor, $1 \times S G$ tonic & & foc. cryp. \\
\hline & III:28 & $\mathrm{M}$ & $6.0^{\circ}$ & 24 & 32 & DZP & TCS & & BFIC \\
\hline & IV:1 & $\mathrm{F}$ & 6.6 & 12.6 & 5 & VPA/PHB & atonic, GTCS & & BFIC \\
\hline & IV:2 & $\mathrm{M}$ & 5.0 & 18.0 & 2.8 & $\mathrm{PHB} / \mathrm{VPA}$ & tonic, atonic & & BFIC \\
\hline & IV:15 & $\mathrm{F}$ & 3.1 & 3.3 & 0.8 & VPA & tonic, GTCS & & BFIC \\
\hline 3 & II:1 & $\mathrm{F}$ & 5.1 & 5.5 & 15 & VPA & tonic, atonic & PKD & ICCA \\
\hline & II:2 & $\mathrm{F}$ & 5.0 & 5.4 & 13 & $\mathrm{PHB} / \mathrm{VPA}$ & $\begin{array}{l}\text { tonic, atonic, jerks around } \\
\text { mouth }\end{array}$ & PKD & ICCA \\
\hline 4 & $\mathrm{I}: 1$ & M & $6-7$ & 24 & 64 & none & GTCS & & BFIC \\
\hline & II:2 & $\mathrm{F}$ & 9.0 & 18 & 36 & none & GTCS & & BFIC \\
\hline & II:3 & $\mathrm{M}$ & 9.0 & 24 & 33 & none & GTCS & & BFIC \\
\hline & III:1 & M & 9.9 & 25.4 & 8 & VPA & GTCS & & BFIC \\
\hline & III:2 & $\mathrm{M}$ & 6.3 & 9.9 & 3 & none & GTCS & & BFIC \\
\hline & III:3 & $\mathrm{M}$ & 1 day & 2 days & 1.7 & PHB & GTCS & & RS \\
\hline 5 & II:4 & $\mathrm{F}$ & 8.0 & 15 & 67 & $?$ & flabby, unconscious & & BFIC \\
\hline & III:8 & $\mathrm{F}$ & 8.0 & 18 & 43 & PHB & atonic, GTCS & & BFIC \\
\hline & III:9 & $\mathrm{F}$ & none & & 40 & none & & PKD & PKD \\
\hline & III:11 & $\mathrm{F}$ & 5.0 & 22 & 39 & PHB & atonic, jerks around mouth & PKD & ICCA \\
\hline & III:14 & $\mathrm{M}$ & 5.2 & 33 & 31 & PHB & atonic, jerks around mouth & PKD & ICCA \\
\hline & IV:7 & M & 4.9 & 36 & 12 & none & GTCS & & BFIC \\
\hline & IV:8 & M & 4.9 & 5.4 & 8 & none & GTCS & & BFIC \\
\hline & IV:10 & $\mathrm{F}$ & 4.6 & 12.7 & 13 & CBZ & CPS & & BFIC \\
\hline & IV:13 & $\mathrm{F}$ & 7.0 & 8.6 & 4 & VPA & GTCS, tonic & & BFIC \\
\hline 6 & $\mathrm{I}: 1$ & M & 3.0 & 6 & $45^{\dagger}$ & none & GTCS & FHM & BFIC \\
\hline & II:1 & $\mathrm{M}$ & 5.0 & 9 & 44 & none & gazing, chewing & & BFIC \\
\hline & II:4 & $\mathrm{F}$ & 5.0 & 15 & 41 & PHT & GTCS +8 yrs: $1 \times$ GTCS & FHM & BFIC \\
\hline & II:5 & $\mathrm{F}$ & 3.0 & 3 & 40 & none & $1 \times$ GTCS & FHM & BFIC \\
\hline & II:7 & $\mathrm{M}$ & 1.5 & 4 & 31 & $?$ & GTCS & FHM & BFIC \\
\hline & III:3 & $\mathrm{F}$ & 3.8 & 8.5 & 12 & VPA & GTCS & ACA & BFIC \\
\hline 7 & II:1 & M & $?$ & $<5$ yrs & 67 & $?$ & $?$ & & BFIC? \\
\hline & III:5 & $\mathrm{F}$ & 5.1 & 6.9 & 35 & PHB & GTCS & & BFIC \\
\hline & III:8 & $\mathrm{M}$ & $4-5$ & 15 & 35 & PHB & TCS & & BFIC \\
\hline & III:11 & $\mathrm{F}$ & $4-5$ & 11 & 30 & none & TCS & & BFIC \\
\hline & III:13 & $\mathrm{F}$ & 9.1 & 11 & 24 & PHB & TCS & & BFIC \\
\hline & III:15 & $\mathrm{F}$ & 9.5 & 11 & 32 & PHB & GTCS & & BFIC \\
\hline & III:17 & $\mathrm{F}$ & 9.0 & 11 & 29 & PHB & $1 \times$ GTCS, tonic & & BFIC \\
\hline & III:19 & $\mathrm{F}$ & 4.0 & 14 & 19 & PHT & tonic & & BFIC \\
\hline & III:22 & $\mathrm{F}$ & 9.4 & $?$ & 32 & none & GTCS & & BFIC \\
\hline & IV:1 & $\mathrm{F}$ & 6.0 & 11 & 7 & none & atonic, GTCS & & BFIC \\
\hline & IV:2 & $\mathrm{M}$ & 5.2 & 5.3 & 11 & VPA & GTCS & & BFIC \\
\hline & IV:4 & $\mathrm{M}$ & 5.7 & 5.8 & 1.8 & VPA & atonic, tonic, GTCS & & BFIC? \\
\hline & IV:8 & $\mathrm{F}$ & 14.0 & 14 & 5 & none & flabby, groggy & & $?$ \\
\hline
\end{tabular}

$\mathrm{M}=$ male, $\mathrm{F}=$ female, yrs = years, $\mathrm{a}=$ still has seizures, ${ }^{\dagger}=$ age at death, $?=$ unknown. AEDs = anti-epileptic drugs, $\mathrm{PHB}=$ phenobarbital, $\mathrm{VPA}=$ valproic acid, $\mathrm{PHT}=$ phenytoin, $\mathrm{LTG}=$ lamotrigine, $\mathrm{CBZ}=$ carbamazepine, $\mathrm{VGB}=\mathrm{vigabatrin}, \mathrm{DZP}=$ diazepam, $(\mathrm{G}) \mathrm{TCS}=$ (generalized) tonic-clonic seizures, $\mathrm{CPS}=$ complex partial seizures, $\mathrm{SG}=$ secondary generalized. Other $=$ other symptoms, $\mathrm{PKD}=$ paroxysmal kinesigenic dyskinesias, $\mathrm{FHM}=$ familial hemiplegic migraine, $\mathrm{ACA}=$ acute cerebellar ataxia. Diagnosis: $\mathrm{BFIC}=$ benign familial infantile convulsions, ICCA $=$ BFIC + PKD, foc. cryp. = focal cryptogenic, $\mathrm{RS}=$ remote symptomatic. 


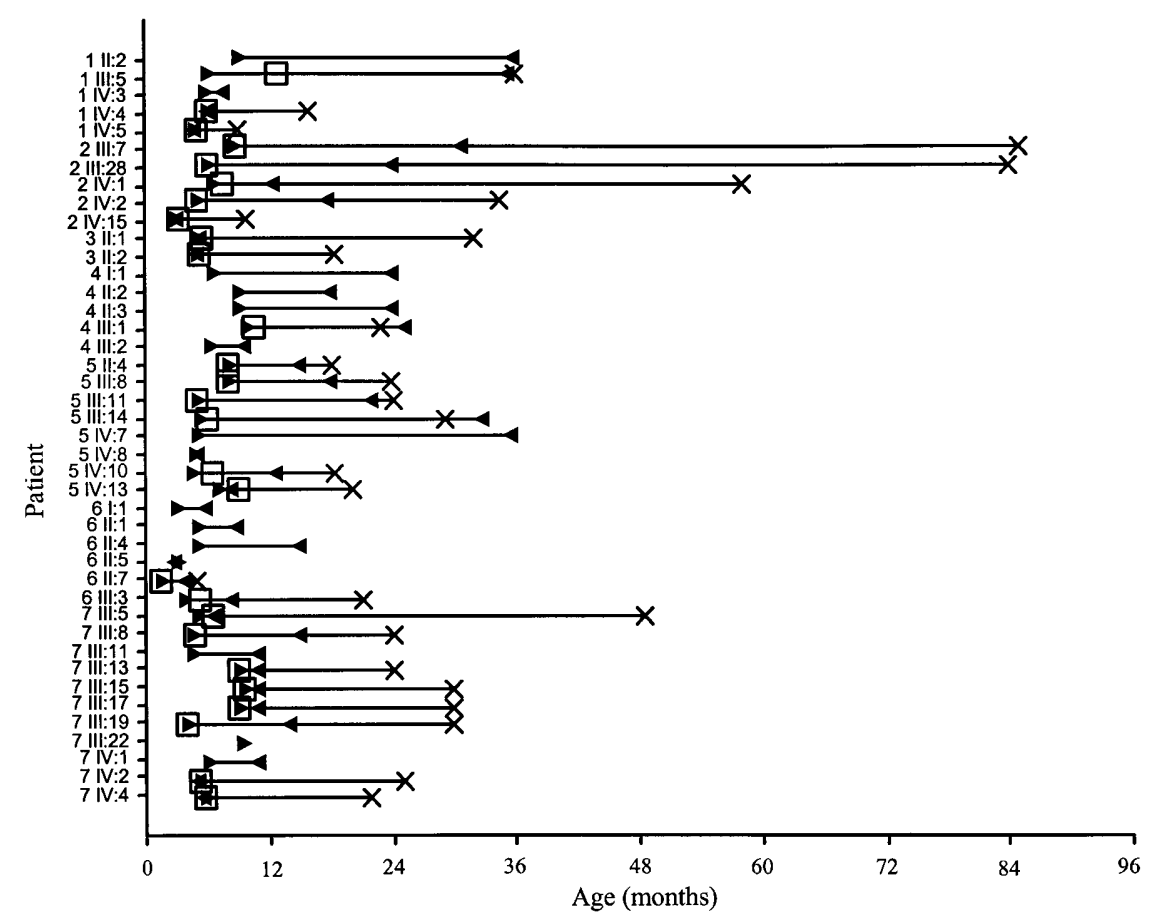

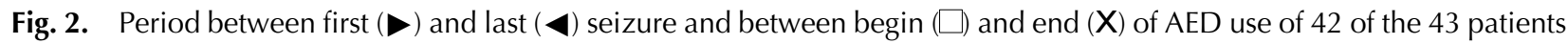
with infantile convulsions (no details about the epilepsy of individual II:1 of family 7 were available). For the patients that were still using AEDs at the time of evaluation, the end of AED has been set at the age at evaluation. seizures during a very short period. Therefore, the symbols of first and last seizure overlap.)

Seizures were reported to occur in clusters (several seizures in one or a number of consecutive days) in $22(51 \%)$ of the patients with BFIC. Some patients experienced only a few seizures, and one patient experienced a single seizure (family 6 , II:5).

An EEG was performed in 26 of the $43(60 \%)$ patients with infantile convulsions, and of another six patients it was unknown whether an EEG was recorded. Most interictal EEGs were reported not to show any abnormalities. In one patient (family 2 , IV:2, Fig. 3), however, bilateral synchronous spikewave like complexes were observed with frontotemporal maximum, and in one patient the interictal EEG was too slow with isolated bitemporal spikewave complexes, right more than left (family 7, IV:4, Fig. 4).

One interictal EEG of another patient (family 5, IV:10) was reported to show right temporal spikes, polyspikes and spike-wave complexes; the EEG recording was, however, destroyed and could, therefore, not be reviewed. An ictal EEG was recorded in two patients with infantile convulsions, showing temporal spike-wave complexes (family 3, II:1, recordings were destroyed and could not be reviewed), and secondary generalized spike-wave complexes with left parietocentral and right frontotemporal foci (family 2, IV:15, Fig. 5). A CT was performed in seven patients with infantile convulsions, showing no abnormalities. The MRI in another patient (family 7, IV:4) showed delayed opercularization of unknown cause.

Of the 43 patients with infantile convulsions, 14 $(33 \%)$ were not treated with anti-epileptic drugs. Phenobarbital (PHB) and valproic acid (VPA) were most often prescribed in the other patients with BFIC (Table 1). Seizures remitted shortly after the initiation of AEDs in nine of the 11 children that were treated with VPA (one other child (family 3, II:2) started VPA after seizure remission because of restlessness), and in six of the 13 children that were treated with PHB. In almost all children AEDs were withdrawn before the age of 3 years (Fig. 2). Two children experienced seizures during initial withdrawal of AEDs around the age of 1 year (family 7, III:15 and III:19), after which AEDs were continued until later age. One other patient experienced one seizure after withdrawal of AEDs at the age of 23 months that was possibly provoked by fever (family 4, III:1), but AEDs were not restarted.

Subsequent psychomotor development was normal in all but one of the patients. The patient with MRI abnormalities (family 7, IV:4, age 22 months) had a mild developmental delay with signs of visual field deficits, probably as a result of the status epilepticus he experienced. 

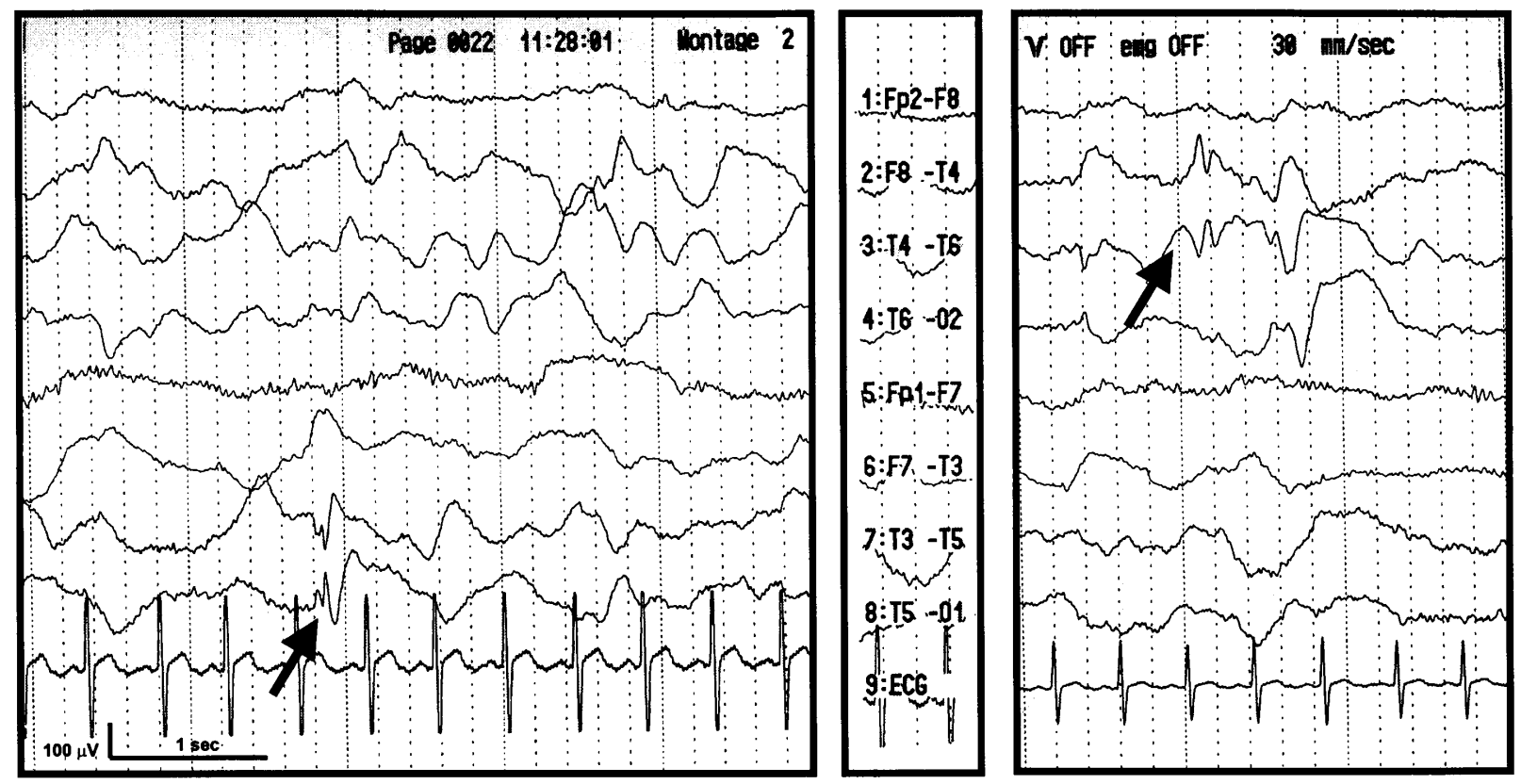

Fig. 4. Interictal EEG of patient IV:4 of family 7 at age 6 months. The background was too slow and isolated bitemporal spike-wave complexes were observed.

In two of our families (3 and 5), paroxysmal kinesigenic dyskinesias were present in some relatives with onset in childhood or early adolescence. All but one of these patients had also suffered from infantile convulsions. They experienced short periods (up to one minute) of dystonia of their arm(s) and/or legs with contortion of their face, triggered by walking/running, standing up, walking the stairs or stress. The frequency of these dyskinesias decreased over time in all patients.

In family 7, no linkage was observed with one of the known loci for BFIC on chromosome 2q24, $16 \mathrm{p} 12-\mathrm{q} 12$, or $19 \mathrm{q}$, but linkage could not definitively be excluded for chromosome 16p (data not shown). ${ }^{9-11}$ Linkage analysis was not performed in the other families since they were probably too small to confirm or exclude linkage to one of the loci.

\section{Discussion}

We describe the clinical characteristics of seven families with BFIC, of which three are multigenerational with affected relatives in several branches.

The phenotypes in our families were practically similar to those in other described families with BFIC, but there were also some minor differences (see appendix at the end of this article). ${ }^{1-6,8}$ Most authors described a unilateral onset of jerks, something that could not be confirmed in all of our families, probably because of the lack of detailed information. On the other hand, Echenne et al. reported that seizures in their families were generalized instead of having a focal onset (based on two interictal EEG reports that were not reviewed by themselves). ${ }^{5}$ Ictal EEGs were, however, not recorded in their patients, which suggests that seizures in their families might also have been secondary generalized.

Furthermore, Vigevano et al. observed ictal EEG abnormalities in the occipitoparietal areas with secondary generalization in five children. ${ }^{2}$ Ictal EEGs in our families showed temporal spike-wave complexes in one patient and secondary generalized spike-wave complexes with onset in the paracentral and frontotemporal areas in another patient. Since in both studies few ictal EEG recordings were available, and no other ictal EEGs have been described in families with BFIC, EEG abnormalities might be more variable than reported by Vigevano et al. ${ }^{2}$ Indeed, Watanabe et al. described EEG abnormalities initiating in the temporal, central, frontal, or parietal area in nine children with benign infantile convulsions. ${ }^{1}$ Three of these children had a positive family history for epilepsy at infantile age. The sites of initial paroxysmal discharges on the ictal EEG in these children were also variable.

Since one child had already experienced his first seizure at the age of 6 weeks, the onset of BFIC might also be earlier in life than reported until now. ${ }^{2,3}$ 
The International League Against Epilepsy has identified three idiopathic syndromes with onset in the first year of life: (1) benign familial neonatal convulsions (BFNC), (2) benign neonatal convulsions (BNC), and (3) benign myoclonic epilepsy (BME). ${ }^{18}$ BFNC and BNC are characterized by clonic and apnoeic seizures with onset in the 1st week of life and remission usually before the 6th month of life. BME has its onset around the age of 1 year and is characterized by brief bursts of generalized myoclonus. Distinction between these three syndromes and BFIC can be made on basis of differences in age at onset and seizure type.

Mutations in two genes encoding voltage-gated potassium channels have been found to be responsible for BFNC. ${ }^{19-21}$ Other epilepsy syndromes have been shown to be caused by mutations in genes encoding ion channels as well.22-29 Our families showed autosomal dominant inheritance with high penetrance. The described loci on chromosome $19 q$ and $2 \mathrm{q} 24$ were found not to be responsible for the epilepsy in family 7 (data not shown), but linkage could not definitively be excluded for chromosome 16p12-q12.9-11 Further studies with additional markers in this region will hopefully provide us with more information.

Two obligate carriers of family 2 had epilepsy with later onset, as well as one other relative without offspring. All three patients did not experience infantile convulsions. Only a few families with infantile convulsions have been described where seizures occurred at later age in some relatives; most were families with infantile convulsions and paroxysmal dyskinesias. ${ }^{8,13,15}$ Whether the patients in our family show another phenotypic expression of BFIC or are phenocopies has to be determined with genetic studies. In three of our families, the epilepsy was associated with other paroxysmal disorders: kinesigenic dyskinesias in two and familial hemiplegic migraine in another. Many paroxysmal disorders, such as familial hemiplegic migraine, have been found to be caused by mutations in ion channels, confirming that paroxysmal disorders, including epilepsy, are channelopathies. ${ }^{30}$ The co-occurrence of epilepsy and episodic ataxia type 1 led to the conclusion that epilepsy and (some) paroxysmal movement disorders might have the same underlying mechanism. . $^{31-33}$

We encountered several difficulties in classifying the BFIC-syndrome. First, parents are needed to obtain the medical history of their affected child, especially in the older generations whose medical information about infantile convulsions has already been destroyed by the hospitals. Recollection of the symptoms by the parents is often poor.
For instance, other studies reported that patients had unilateral limb jerks, later becoming bilateral. $^{2,8}$ This was not reported in our families, because most parents could not recollect whether the tonic-clonic seizures started unilaterally or were generalized at onset. Since all the observed EEG abnormalities had a focal onset, we think that our families suffered from secondary generalized epilepsy. Second, BFIC concerns a relatively mild epilepsy syndrome that is often not recognized as epilepsy by parents or physician. Third, parents often do not consult a paediatrician or neurologist because of the mildness of the syndrome, thus no data of EEGs or other examinations are available. Even if EEG results are available, they are often inconclusive because interictal EEGs usually do not show any abnormalities. So again, the seizures may not be recognized as epilepsy. Fourth, the parents may miss seizures if they rarely occur. The observed incomplete penetrance in families 2 and 7 may, therefore, be false: it is possible that seizures occurred, but were just not witnessed.

In conclusion, benign familial infantile convulsions are benign seizures, stereotyped in their age at onset and their symptoms. We observed, however, that physicians (general practitioners, paediatricians and neurologists) might not recognize infantile convulsions as epilepsy. To give parents reassurance and more certainty about the disease of their child, we would, therefore, like to emphasize that, despite the sometimes low frequency of seizures and the fact that most interictal EEGs do not show any abnormalities, infantile convulsions are epilepsy. Seizures often remit shortly after the initiation of AEDs but, because of the mildness of the syndrome and the spontaneous remission of seizures, patients with low seizure frequency do not necessarily have to be treated. Since none of the children experienced any seizures after the age of 3 years, we think that AEDs can probably be withdrawn at least at that age or after 1 or 2 years of seizure freedom.

\section{Acknowledgements}

We would like to thank the families for their cooperation. PMC Callenbach is a research fellow supported by a grant from the Netherlands Organization for Scientific Research (NWO, 94033-030) and the National Epilepsy Fund (98-14).

\section{References}

1 Watanabe K, Yamamoto N, Negoro T et al. Benign complex partial epilepsies in infancy. Pediatr Neurol 1987; 3: 208-211. 
2 Vigevano F, Fusco L, Di Capua M et al. Benign infantile familial convulsions. Eur J Pediatr 1992; 151: 608-612.

3 Lee WL, Low PS, Rajan U. Benign familial infantile epilepsy. J Pediatr 1993; 123: 588-590.

4 Malafosse A, Beck C, Bellet $\mathrm{H}$ et al. Benign infantile familial convulsions are not an allelic form of the benign familial neonatal convulsions gene. Ann Neurol 1994; 35: 479-482.

5 Echenne B, Humbertclaude V, Rivier F, Malafosse A, Cheminal R. Benign infantile epilepsy with autosomal dominant inheritance. Brain Dev 1994; 16: 108-111.

6 Giordano L, Accorsi P, Valseriati D et al. Benign infantile familial convulsions: natural history of a case and clinical characteristics of a large Italian family. Neuropediatrics 1999; 30: 99-101.

7 Gennaro E, Malacarne M, Carbone I et al. No evidence of a major locus for benign familial infantile convulsions on chromosome 19q12-q13.1. Epilepsia 1999; 40: 1799-1803.

8 Baralle D, Dearlove AM, Beach R, Ffrench-Constant C, Reid E. Benign familial infantile convulsions: report of a UK family and confirmation of genetic heterogeneity. J Med Genet 2000; 37: E31-E33.

9 Guipponi M, Rivier F, Vigevano F et al. Linkage mapping of benign familial infantile convulsions (BFIC) to chromosome 19q. Hum Mol Genet 1997; 6: 473-477.

10 Caraballo R, Pavek S, Lemainque A et al. Linkage of benign familial infantile convulsions to chromosome 16p12-q12 suggests allelism to the infantile convulsions and choreoathetosis syndrome. Am J Hum Genet 2001; 68: 788-794.

11 Malacarne M, Gennaro E, Madia F et al. Benign familial infantile convulsions: mapping of a novel locus on chromosome $2 q 24$ and evidence for genetic heterogeneity. Am J Hum Genet 2001; 68: 1521-1526.

12 Szepetowski P, Rochette J, Berquin P et al. Familial infantile convulsions and paroxysmal choreoathetosis: a new neurological syndrome linked to the pericentromeric region of human chromosome 16 . Am J Hum Genet 1997; 61: 889-898.

13 Lee WL, Tay A, Ong HT et al. Association of infantile convulsions with paroxysmal dyskinesias (ICCA syndrome): confirmation of linkage to human chromosome 16p12-q12 in a Chinese family. Hum Genet 1998; 103: 608-612.

14 Tomita H, Nagamitsu S, Wakui K et al. Paroxysmal kinesigenic choreoathetosis locus maps to chromosome 16p11.2-q12.1. Am J Hum Genet 1999; 65: 16881697.

15 Swoboda KJ, Soong B, McKenna C et al. Paroxysmal kinesigenic dyskinesia and infantile convulsions: clinical and linkage studies. Neurology 2000; 55: 224-230.

16 Terwindt GM, Ophoff RA, Lindhout D et al. Partial cosegregation of familial hemiplegic migraine and a benign familial infantile epileptic syndrome. Epilepsia 1997; 38: 915-921.

17 Commission on Classification and Terminology of the International League Against Epilepsy. Proposal for revised clinical and electroencephalographic classification of epileptic seizures. Epilepsia 1981; 22: 489-501.
18 Commission on Classification and Terminology of the International League Against Epilepsy. Proposal for revised classification of epilepsies and epileptic syndromes. Epilepsia 1989; 30: 389-399.

19 Singh NA, Charlier C, Stauffer D et al. A novel potassium channel gene, $\mathrm{KCNQ}$, is mutated in an inherited epilepsy of newborns. Nature Genet 1998; 18: 25-29.

20 Biervert C, Schroeder BC, Kubisch C et al. A potassium channel mutation in neonatal human epilepsy. Science 1998; 279: 403-406.

21 Charlier C, Singh NA, Ryan SG et al. A pore mutation in a novel KQT-like potassium channel gene in an idiopathic epilepsy family. Nature Genet 1998; 18: 53-55.

22 Steinlein OK, Mulley JC, Propping $\mathrm{P}$ et al. A missense mutation in the neuronal nicotinic acetylcholine receptor alpha 4 subunit is associated with autosomal dominant nocturnal frontal lobe epilepsy. Nature Genet 1995; 11: 201-203.

23 De Fusco M, Becchetti A, Patrignani A et al. The nicotinic receptor beta2 subunit is mutant in nocturnal frontal lobe epilepsy. Nature Genet 2000; 26: 275-276.

24 Phillips HA, Favre I, Kirkpatrick M et al. CHRNB2 is the second acetylcholine receptor subunit associated with autosomal dominant nocturnal frontal lobe epilepsy. Am J Hum Genet 2001; 68: 225-231.

25 Wallace RH, Wang DW, Singh R et al. Febrile seizures and generalized epilepsy associated with a mutation in the $\mathrm{Na}+$-channel beta1 subunit gene SCN1B. Nat Genet 1998; 19: 366-370.

26 Escayg A, MacDonald BT, Meisler $\mathrm{MH}$ et al. Mutations of SCN1A, encoding a neuronal sodium channel, in two families with GEFS+2. Nat Genet 2000; 24: 343-345.

27 Baulac S, Huberfeld G, Gourfinkel-An I et al. First genetic evidence of $\mathrm{GABA}(\mathrm{A})$ receptor dysfunction in epilepsy: a mutation in the gamma2-subunit gene. Nature Genet 2001; 28: 46-48.

28 Wallace $\mathrm{RH}$, Marini C, Petrou S et al. Mutant GABA(A) receptor gamma2-subunit in childhood absence epilepsy and febrile seizures. Nature Genet 2001; 28: 49-52.

29 Jouvenceau A, Eunson LH, Spauschus A et al. Human epilepsy associated with dysfunction of the brain P/Q-type calcium channel. Lancet 2001; 358: 801-807.

30 Felix R. Channelopathies: ion channel defects linked to heritable clinical disorders. J Med Genet 2000; 37: 729-740.

31 Zuberi SM, Eunson LH, Spauschus A et al. A novel mutation in the human voltage-gated potassium channel gene (Kv1.1) associates with episodic ataxia type 1 and sometimes with partial epilepsy. Brain 1999; 122 ( Pt 5): 817-825.

32 Berkovic SF. Paroxysmal movement disorders and epilepsy: links across the channel. Neurology 2000; 55: 169-170.

33 Eunson LH, Rea R, Zuberi SM et al. Clinical, genetic, and expression studies of mutations in the potassium channel gene KCNA1 reveal new phenotypic variability. Ann Neurol 2000; 48: 647-656. 


\section{Appendix: Case histories}

Family 1

The proband of this family (IV:5) presented in the hospital at the age of 4.7 months with seizures with unresponsiveness, deviation of her head to the right, staring, and sometimes jerks of her left leg. She also suffered from one generalized tonic-clonic seizure. Seizures lasted up to 5-10 minutes, and occurred up to three times daily. A post-ictal EEG was asymmetrical to the detriment of the left hemisphere with fast activity in the right hemisphere and slow theta-frequency with a frontotemporal maximum in the left hemisphere. She was treated with valproic acid and seizures resolved 3 days later. The EEG did not show any abnormalities anymore, brain imaging was not performed. Until now (age 9 months), psychomotor development has been normal. She is still using valproic acid.

Her paternal grandfather (II:2, age 69 years) of normal intelligence suffered from numerous tonicclonic seizures with deviation of the eyes between approximately 9 months and 3 years of age. It is unknown whether he was treated with AEDs.

Her 31-year old father (III:5) experienced short seizures with unresponsiveness and deviation of the eyes since the age of 6 months. At the age of 1 year, he had a tonic-clonic seizure with cyanosis and foam around his mouth, during which he fell down the stairs. After the seizure his right arm and leg were paralysed for a short period. Later that day, he experienced another tonic-clonic seizure lasting approximately 5 minutes. $\mathrm{He}$ received phenobarbital, and experienced approximately one short seizure per month until the age of approximately 3 years. The interictal EEG did not show any abnormalities, brain imaging was not performed. Subsequent development was normal.

Her sister (IV:3) experienced one atonic seizure at the age of 6 months during baby swimming with unresponsiveness, cyanosis, and deviation of the head and eyes. She also experienced small seizures with deviation of the eyes until the age of 8 months. The EEG showed no abnormalities and she was not treated with AEDs. Psychomotor development has been normal until now (age 5 years).

Her brother (IV:4) experienced clusters of tonic and atonic seizures with deviation of the eyes, cyanosis, and jerks around his mouth lasting approximately 8 minutes at 6 months of age. He was successfully treated with valproic acid until the age of 16 months, and subsequent development has been normal until now (age 4 years). The interictal EEG and CT showed no abnormalities.
Family 2

The proband of this family (IV:1) had her first seizure at the age of 6.6 months: she started moaning, gazed, was unresponsive, and became atonic and cyanotic for approximately 2 minutes, occurring up to three times daily. A few weeks later, she also had tonic-clonic seizures with cyanosis. The interictal EEG and CT showed no abnormalities. Valproic acid was not successful, but seizures remitted after the start of phenobarbital, which was continued until the age of 4.7 years. At the age of 1 year, she had one last, possibly febrile, seizure. Psychomotor development has been normal until now (age 5 years).

Her father (III:1), who has normal intelligence, experienced two or three seizures at the age of 31 years with unconsciousness, jerking of both arms, making sounds, and incontinence. The interictal EEG and CT were normal. He has never been treated with AEDs. Currently (age 38 years), he experiences short periods of decreased vision, derealization, disorientation, and confusion a few times yearly.

Her grandfather (II:1) had a few seizures at the age of 17 years, during which he became unconscious and atonic or had tonic-clonic jerks. From the age of 59 years onwards, he experienced tonicclonic seizures and complex partial seizures with lowered consciousness, deviation of the eyes, disorientation, and sometimes paraesthesiae in the fingers and cheeks, or olfactory and auditory symptoms. The interictal EEG did not show any abnormalities. He was alternately treated with phenytoin, valproic acid, and lamotrigine with variable effect. At age 59 years, an acusticus neurinoma was surgically removed. Currently (age 69 years), he experiences complex partial seizures approximately once a month; the last tonic-clonic seizure occurred at age 66. He is now prescribed lamotrigine monotherapy.

A 30-year old aunt (III:7) experienced clusters of short tonic-clonic seizures between 8.5 and approximately 31 months of age. All interictal EEGs were normal, no brain imaging was performed. She was successfully treated with phenobarbital and phenytoin until the age of approximately 7 years. Psychomotor development has been normal.

The probands' younger brother (IV:2) experienced a cluster of 2 days with several tonic seizures (left more than right) at the age of 5 months. Furthermore, he experienced short atonic seizures with unresponsiveness, cyanosis, and deviation of the eyes. The interictal EEG showed bilateral synchronous spike-wave like complexes with frontotemporal maximum (Fig. 3). The CT showed 
no abnormalities. Phenobarbital was started during the first cluster, but he kept infrequent seizures. Phenobarbital was, therefore, replaced by valproic acid, and seizures remitted at the age of 18 months. Subsequent psychomotor development has been normal until now (age 2.8 years). He is still on valproic acid monotherapy.

A 25-year old cousin of the father of the proband (III:10) with normal intelligence experienced simple partial seizures between 16.7 and 18.8 years of age with paraesthesiae and myoclonic jerks in one hand, with decreasing duration and frequency over time. The first seizure was secondary generalized, during which he became unconscious, tonic, was incontinent, and had foam around his mouth. The EEG after the first seizure showed left temporal sharp complexes (alpha and alpha-theta), spreading to left frontolateral. The CT showed no abnormalities. He was treated with carbamazepine and vigabatrin until the age of 20 years.

Another cousin of her father (III:28) suffered from seizures between approximately 6 and 24 months of age lasting 5 minutes with unconsciousness, cyanosis, deviation of the eyes and tonicclonic jerks in the arms. Over time, frequency and duration diminished. CT and MRI were not performed, results of EEGs have been destroyed. He was treated with diazepam until the age of approximately 7 years and has a normal intelligence (current age 32 years).

The daughter of this cousin (IV:15) presented in the hospital at the age of 3 months with clusters of tonic seizures with unconsciousness, smacking, deviation of the eyes, and jerks around mouth and eyes and in the arms. A few days later, she suffered from tonic-clonic seizures with deviation of the eyes and head, and cyanosis. The ictal EEG showed secondary generalized spike-wave complexes with left parietocentral and right frontotemporal foci (Fig. 5). CT and MRI were not performed. She became seizure-free directly after starting valproic acid, and subsequent development has been normal until now (age 9.5 months). She still uses valproic acid.

\section{Family 3}

The proband of this family (II:2) presented in the hospital at the age of 5 months with a cluster of three tonic and atonic seizures in 1 day with deviation of the eyes, unresponsiveness, cyanosis, thrusting breathing, and slight jerks around her mouth; 1 week later, she had another cluster with three similar seizures. The interictal EEG and CT did not show any abnormalities. She was treated with phenobarbital, and seizures remitted within 5 days. The phenobarbital was later replaced by valproic acid because of restlessness, and she was treated until the age of 18 months. From the age of approximately 6 years, she experienced dystonia of both arms, preceded by an upward moving sensation starting in both feet that was induced by the initiation of walking, lasting approximately one minute. The dystonia was successfully treated with carbamazepine. Currently (age 13 years), she only experiences the sensation in her feet if she refrains from carbamazepine, but dystonia has not recurred.

Her older sister (II:1) suffered from clusters of 4-8 tonic and atonic seizures per day with unresponsiveness, deviation of the eyes, irregular breathing, and sometimes vomiting afterwards at the age of 5 months that were successfully treated with valproic acid until the age of 32 months. The ictal EEG was reported to show temporal spikewaves, the interictal EEG and CT showed no abnormalities. The ictal EEG was, however, destroyed, and could not be reviewed by us. From the age of approximately 9 years, she experienced dystonia and choreoathetosis of one arm (alternating left and right) accompanied by involuntary movements of her mouth that was preceded by the same sensation in one foot as her sister felt, occurring up to three times daily. Currently (age 15 years), she does not suffer from dystonia or choreoathetosis anymore, but sometimes experiences the sensations in her feet. Both girls have normal intelligence. No other persons in this family suffered from seizures or dystonia.

\section{Family 4}

The proband of this family (III:2) experienced his first tonic-clonic seizure at the age of 6 months, during which he was unconscious, became red, drooled, and his eyes deviated. He experienced four seizure episodes, always occurring in clusters of 1 or 2 days, until remission at the age of 10 months. The interictal EEG showed no abnormalities, brain imaging was not performed. He was not treated with AEDs. Psychomotor development has been normal until now (current age 3 years).

His older brother (III:1) suffered from several clusters of two days with tonic-clonic seizures between 10 and 11 months of age with jerks in the arms and sometimes also the legs, deviation of the eyes and redness. The interictal EEG showed no abnormalities, CT and MRI were not performed. He was successfully treated with valproic acid until the age of 23 months; 2 months after AED 
withdrawal he experienced one last seizure that was possibly provoked by fever, but AEDs were not restarted. Psychomotor development has been normal until now (age 8 years).

The 36-year old mother of the two boys (II:2) had a few tonic-clonic seizures between the age of 9 and 18 months. The interictal EEG showed no abnormalities, and she was not treated with AEDs. Brain imaging was not performed. She has normal intelligence.

Their 33-year old uncle (II:3) experienced tonicclonic seizures between the age of 9 months and approximately 2 years, for which he was not treated with AEDs. The interictal EEG showed no abnormalities, brain imaging was not performed. Subsequent development was normal.

Their grandfather (I:1, age 64 years) probably had the same seizures as his children between the age of 6-7 months and 2 years, but details are missing. He was not treated with AEDs and has normal intelligence.

Their cousin (III:3) was delivered via vacuum extraction and had a perinatal asphyxia with multiorgan failure and a plexus brachialis lesion. One day after birth, he had two symptomatic generalized tonic-clonic seizures for which he was treated with phenobarbital until the age of 5 months. The MRI showed an extracerebral bleeding due to the vacuum extraction. Currently (age 21 months), he shows normal psychomotor development. Seizures have not re-occurred.

\section{Family 5}

The proband of this family (IV:13) suffered from her first seizure at 7 months of age, 15 minutes after feeding: her eyes deviated, she became unconscious and showed tonic-clonic jerks for approximately 2 minutes. Afterwards, she vomited and slept for some hours. In total, she suffered from three seizures (tonic and tonic-clonic). The interictal EEG showed no abnormalities, CT and MRI were not performed. She became seizure-free after the start of valproic acid, and psychomotor development was normal until now (age 4 years). Valproic acid was discontinued at age 20 months.

One of her sisters (IV:10) suffered from clusters of seizures between 5 and 13 months of age, with sometimes trembling of mouth and eyes, cyanosis, deviation of the eyes and head, and unresponsiveness for up to 1 minute. One interictal EEG was reported to show right temporal spikes, polyspikes, and irregular spike-wave complexes. This EEG had, however, already been destroyed and could, therefore, not be reviewed. The other EEG recordings, CT, and screening for metabolic disorders showed no abnormalities. The girl was treated with carbamazepine until the age of 18 months, and had a normal psychomotor development (current age 13 years).

Their 67-year old grandmother (II:4) of normal intelligence suffered from seizures between the age of approximately 8 and 15 months, during which she was flabby and unconscious. It is unknown whether she had tonic-clonic jerks. No EEG, CT, or MRI was performed. She was treated with AEDs until the age of approximately 18 months.

The mother of the two girls (III:11) suffered from clusters of atonic seizures with clonic jerks around the mouth for a few minutes, paleness, deviation of the eyes, and unconsciousness between approximately 5 and 22 months of age. EEG, CT, and MRI were not performed. Seizures remitted with phenobarbital (treatment until the age of 2 years). Psychomotor development was normal. Around puberty, she started to experience short contortions of her arms and mouth when she quickly stood up or went up the stairs. Currently (age 39 years), she does not have any symptoms.

A 43-year old aunt (III:8) of normal intelligence suffered from atonic and tonic-clonic seizures with deviation of the eyes and unconsciousness between approximately 8 and 18 months of age. The interictal EEG showed no abnormalities, CT and MRI were not performed. Seizures remitted with phenobarbital (treatment until the age of 2 years).

The oldest son of this aunt (IV:7, age 12 years) of normal intelligence experienced seizures between the age of 5 months and approximately 3 years. Seizures were generalized tonic-clonic with unconsciousness, deviation of the eyes, and cyanosis, and occurred in clusters with decreasing frequency over time. The interictal EEG did not show any abnormalities, CT and MRI were not performed. No AEDs were prescribed.

His younger brother (IV:8, age 8 years) experienced a cluster of well over 1 week with several afebrile tonic-clonic seizures at the age of 5 months. The frequency of seizures decreased during this week. EEG, CT, and MRI were not performed, and no AEDs were prescribed. Subsequent development has been normal.

Another aunt (III:9) of normal intelligence did not experience infantile seizures, but around puberty, she started to experience a dystonia of the arms and legs, and a contortion of her face for a few seconds on the sudden initiation of running. She was not treated, but currently (age 40 years) does not experience any symptoms anymore because she avoids triggering situations. 
An uncle (III:14) suffered from clusters of atonic seizures from the age of 5 months, during which he did not respond. From the age of 2 years until the age of approximately 33 months, he infrequently experienced nocturnal atonic seizures after busy days with clonic jerks around his mouth and paleness, preceded by tearfulness. EEG, CT, and MRI were not performed. He was treated with phenobarbital until the age of approximately 2.5 years. Currently (age 31 years), he sometimes experiences short periods with dystonia of his arms and legs with contortion of his face, especially in periods of stress. It is unknown when this started, but frequency has decreased over time.

\section{Family 6}

This family has also been described by Terwindt et al. ${ }^{16}$

The 12-year old proband of this family (III:3) presented in the hospital at age 4 months with periods of gazing, unresponsiveness, paleness, and trembling of arms and legs with sometimes jerks for up to 4 minutes, sometimes occurring in clusters. The interictal EEG showed no abnormalities, CT and MRI were not performed. Since seizure frequency increased over time and sometimes occurred in clusters, valproic acid was started, after which seizures remitted. At the age of 8.5 months, she had one last seizure with staring and trembling for one minute, and valproic acid was discontinued at 21 months of age. Psychomotor development was normal. At the age of 3 and 4 years, she experienced two febrile convulsions, and at the age of 7.5 years, she had a 2-week period of cerebellar ataxia with subacute onset, accompanied by bifrontal and temporal headache and dysarthria. The EEG and CT showed no abnormalities at that time.

Her 41-year old mother (II:4) of normal intelligence experienced tonic-clonic seizures between approximately 5 and 15 months of age, during which she gazed and had jerks of arms and legs. The interictal EEG showed no abnormalities. At the age of 8 years, she had one nocturnal generalized tonic-clonic seizure, and was subsequently treated with phenytoin until the age of 12 years. Furthermore, she suffered from hemiplegic migraine since the age of 10 years.

The probands' grandfather (I:1) was reported to have suffered from clusters of up to 32 tonic-clonic seizures a day between approximately 3 and 6 months of age with twitching and jerking of arms and legs. He was never treated with AEDs.
Furthermore, he had suffered from hemiplegic migraine since early childhood. He died at the age of 45 years of a small cell carcinoma.

The oldest brother of the probands' mother (II:1) experienced clusters of up to 11 seizures a day, during which he gazed and made chewing movements from the age of 5 until the age of 9 months. It is unknown whether an EEG was recorded. He was not treated with AEDs, and had a normal psychomotor development (current age 44 years).

One sister of mother (II:5) experienced one generalized tonic-clonic seizure at the age of 3 months and was never treated with AEDs. It is unknown whether an EEG was recorded. This 40-year old female of normal intelligence also suffered from hemiplegic migraine since the age of 10 years.

Their youngest brother (II:7, age 31 years) experienced several tonic-clonic seizures between the age of 1.5 and approximately 4 months for which he was treated with AEDs for a few months. It is unknown whether an EEG was recorded. Furthermore, he had suffered from hemiplegic migraine since the age of 18 years.

\section{Family 7}

The 22-month old proband of this family (IV:4) experienced a status epilepticus followed by a period of approximately 48 hours with persistent focal seizures at age 6 months. He became unresponsive, atonic, tonic, and pale, with frequently generalized tonic-clonic jerks, and had eyelid myoclonias. The interictal EEG was too slow with isolated bitemporal spike-wave complexes, right more than left (Fig. 4), the MRI showed delayed opercularization of unknown cause. Seizures remitted finally with valproic acid; 3 days after the status epilepticus, he had some signs of cortical blindness, which disappeared spontaneously. Metabolic screening showed no abnormalities. Currently, he has a mild developmental delay with signs of visual field deficits, probably as a result of the status epilepticus. He is still using valproic acid.

His mother (III:13, age 24 years) of normal intelligence had her first seizure at age 9 months, 3 weeks after she fell down the stairs and suffered a concussion. In total, she experienced three tonicclonic seizures of her arms with deviation of the eyes and unconsciousness. She was treated with phenobarbital until the age of approximately 2 years. EEG, CT, and MRI were not performed.

Seizures of an uncle (III:8, age 35 years) of normal intelligence started at age 4-5 months. They were characterized by humming, unresponsiveness, 
deviation of the eyes, protrusion of the tongue, and tonic-clonic jerks of both arms during a few minutes. EEG, CT, and MRI were not performed. He was successfully treated with phenobarbital, and seizures remitted at approximately 15 months of age, after which phenobarbital was withdrawn at the age of 2 years.

A 30-year old aunt (III:11) of normal intelligence suffered from seizures between 4-5 and 11 months of age with the same aspect as III:8, but with shorter duration and lower frequency. She was not treated with AEDs and diagnostic studies were not performed.

A great-uncle (II:1, age 67 years) was said to have seizures before the age of 5 years, but details are missing. None of his brothers and sisters was reported to have had seizures.

A 35-year old cousin of the probands' mother (III:5) had her first seizure at age 5 months, during which she was found unconscious and pale with deviated eyes and tonic-clonic jerks. Because seizure frequency increased over time, she was treated with phenobarbital and seizures remitted. The interictal EEG showed no abnormalities, CT and MRI were not performed. Subsequent development was normal, and phenobarbital was discontinued at the age of approximately 4 years.

The daughter of this cousin (IV:1) experienced atonic and tonic-clonic seizures between six and 11 months of age with deviation of her eyes, occurring once every 1-2 weeks in the morning with decreasing frequency and severity. The interictal EEG showed no abnormalities, no CT or MRI was performed, and she was not treated with AEDs. Psychomotor development was normal (current age 7 years).

The son of one of the sisters of this cousin (IV:2) of normal intelligence suffered from nine generalized tonic-clonic seizures in 2 days at the age of 5 months with deviation of his eyes, thrusting breath, and paleness. The interictal EEG showed no abnormalities, CT and MRI were not performed. He was treated with valproic acid until the age of 2 years, and never experienced seizures anymore (current age 11 years).

Another cousin of the probands' mother (III:15) experienced two tonic-clonic seizures at ages 9.5 and 11 months, lasting 15 minutes with unresponsiveness, deviation of her eyes, and foam around her mouth. EEG results had already been destroyed. She was treated with phenobarbital. The second seizure occurred after withdrawing the phenobarbital at the age of 11 months. The phenobarbital was, therefore, restarted and given until the age of 2.5 years. Subsequent development was normal (current age 32 years).

One of the sisters of this cousin (III: 17) suffered from several tonic seizures and one tonic-clonic seizure between approximately 9 and 11 months of age. She was successfully treated with phenobarbital until the age of 2.5 years, and had a normal psychomotor development (current age 29 years). No EEG, CT, or MRI was performed.

Another sister (III:19) of normal intelligence, aged 19 years, experienced seven tonic seizures between approximately 4 and 14 months of age. The last seizure occurred during the withdrawal of the phenytoin, which was then continued until 2.5 years of age. No EEG, CT, or MRI was performed.

A fifth cousin of mother (III:22, age 32 years) suffered from tonic-clonic seizures that lasted for approximately 10 minutes, with onset at 9 months of age. The doctor had diagnosed the seizures as 'growing pains'. She was never treated, and no additional examinations were performed. It is unknown when she experienced her last seizure. At least two of her brothers and sisters experienced the same, but the parents could not recollect which children had had seizures because these were not recognized as epilepsy. None of them was ever treated, and all had normal intelligence.

The 5-year old daughter of one of the sisters of this cousin (IV:8) experienced three periods of approximately 20 minutes during which she was flabby and groggy with lowered consciousness around the age of 14 months. Physical examination showed no abnormalities, no other investigations were performed. The possibility exists that she was in a postictal state when she was found.

In this family, no linkage was observed with one of the known loci for BFIC on chromosome 2q24, $16 \mathrm{p} 12-\mathrm{q} 12$, or $19 \mathrm{q}$, but linkage could not be excluded completely for chromosome 16p (data not shown). ${ }^{9-11}$ 